\title{
VUV SPECTROSCOPY AND PHOTOCHEMISTRY OF FIVE INTERSTELLAR AND PUTATIVE PREBIOTIC MOLECULES
}

\author{
M. Schwell ${ }^{1}$, F. Gaie-Levrel ${ }^{2}$, Y. Bénilan ${ }^{1}$, M.-C. Gazeau ${ }^{1}$, N. Fray ${ }^{1}$, \\ G. Saul ${ }^{1}$, N. Champion ${ }^{3}$, S. Leach ${ }^{3}$ and J.-C. Guillemin ${ }^{4}$
}

\begin{abstract}
For many years, our group has been investigating the VUV spectroscopy and photochemistry of molecules of astrophysical (Jochims et al. 2006a,b; Leach et al. 2008; Schwell et al. 2012) and prebiotic interest (Schwell et al. 2006). Polyynes and cyano-polyynes that are abundant in the interstellar medium (ISM) and in planetary atmospheres, have been investigated too (e.g. Fray et al. 2010). An aerosol source for reactive and thermo-labile compounds has been developed (Gaie-Levrel et al. 2011) to perform gas-phase measurements. These are necessary to measure intrinsic molecular properties and to compare to quantum chemical calculations. Besides measuring absolute absorption and photoionization cross sections, dissociative channels and their involved excited states are identified for a number of molecules of interstellar interest. Branching ratios of the respective elementary photoreactions are determined in order to understand and model the photochemistry occurring in the ISM. Some very recent results on the dissociative photoionization of methylformate (MF), glycolaldehyde (GA), dimethylether (DIM), aminoacetonitrile (AAC) and cyanoacetylene $(\mathrm{CA})$, are presented here.
\end{abstract}

\section{Results}

Measurements were performed in 2011 in the 9 to $16 \mathrm{eV}$ energy range using the SAPHIRS molecular beam chamber of the DESIRS beamline at the SOLEIL synchrotron radiation facility. SAPHIRS is equipped with a photoelectron/photoion

1 Laboratoire Inter-universitaire des Systèmes Atmosphériques (LISA), UMR CNRS 7583, Universités Paris Est Créteil and Paris Diderot, Institut Pierre Simon Laplace, 61 avenue du Général de Gaulle, 94010 Créteil, France

2 Synchrotron SOLEIL, L'Orme des Merisiers, St. Aubin, BP. 48, 91192 Gif-sur-Yvette Cedex, France

${ }^{3}$ Laboratoire d'Étude du Rayonnement et de la Matière en Astrophysique (LERMA), UMR CNRS 8112, Observatoire de Paris-Meudon, 5 place Jules-Jansen, 92195 Meudon, France

4 Sciences Chimiques de Rennes, École Nationale Supérieure de Chimie de Rennes, CNRS, UMR 6226, Avenue du Général Leclerc, CS 50837, 35708 Rennes Cedex 7, France 
coincidence (PEPICO) spectrometer called DELICIOUS II that combines a velocity map imaging (VMI) system of the photoelectrons with a linear time-of-flight ion mass analyzer (see Garcia et al. 2009). Two modes of operation can be used: 1) From the VMI images, zero kinetic energy electrons can be selected in order to measure Threshold-PEPICO (TPEPICO) mass spectra as a function of the photon energy, with spectral resolutions as small as $1 \mathrm{meV}$. In this mode, vibrational progressions of involved ion states can be studied. 2) In PEPICO energy scans, shown here, no energy selection is applied to the electrons used for the coincidence measurements. However, a maximum kinetic energy $\mathrm{KE}_{\max }$ of the photoelectrons can be chosen by adapting the ion source extraction conditions $\left(\mathrm{KE}_{\max }=6 \mathrm{eV}\right.$ in this study). The spectral resolution, generally lower than in the TPEPICO mode, is given only by the grating monochromator in this case. Ionization cross sections and branching ratios utilizable in photochemical models can only be measured in the PEPICO mode. In this work, spectra up to $15.5 \mathrm{eV}$ have been obtained with about $10 \mathrm{meV}$ resolution. We make use of the gas filter of the beamline which allows for spectrally pure measurements.

1) $\mathrm{MF}\left(\mathrm{HCOOCH}_{3}\right)$ is an important species observed by radioastronomy in hot cores. It is very abundant in star forming regions. Recently, ${ }^{13} \mathrm{C}$ isotopomers have also been observed (Carvajal et al. 2009). Its isomer acetic acid has been studied in the past (Jochims et al. 2006a,b). Experiments on the photochemistry of $\mathrm{HCOOCH}_{3}$ will help to understand the relative abundances of $\mathrm{C}_{2} \mathrm{H}_{4} \mathrm{O}_{2}$ isomers observed in the ISM. Another isomer $\mathrm{GA}\left(\mathrm{HOCH}_{2} \mathrm{CHO}\right)$ is the only sugar detected in the ISM (Halfen et al. 2006). VUV spectra for GA are not found in the literature. For MF, m/z 60 (parent ion), 32, 31, 30, 29, and 15 ions are observed in the 10.8 to $16 \mathrm{eV}$ range. Their appearance energies are determined and compared to earlier TPEPICO work (Nishimura et al. 1987). Although we cannot extract the TPEPICO spectrum since we are using a high $\mathrm{KE}_{\max }$, our values are slightly lower than those of Nishimura et al. (1987). In our measurements, the parent ion is the most intense ion up to about $12.8 \mathrm{eV}$. From this energy on up to $16 \mathrm{eV} \mathrm{m} / \mathrm{z} 31$ $\left(\mathrm{CH}_{3} \mathrm{O}^{+}\right)$is by far the most intense ion. Ion $\mathrm{m} / \mathrm{z} 32$ is weak and the other ions are very weak too.

2) DIM $\left(\mathrm{H}_{3} \mathrm{COCH}_{3}\right)$ is highly abundant in hot cores (see for example Schilke et al. 2001). In the energy range under study we observed $\mathrm{m} / \mathrm{z} 46$ (parent ion), 45, 31, 29 and 15 ions. We were able to determine appearance energies for $\mathrm{m} / \mathrm{z} 46$, 31, and 29. Again, our values are lower than those from earlier work (Butler et al. 1984). The ionization energy (IE) is found at $\mathrm{IE}=(9.96 \pm 0.01) \mathrm{eV}$ and the parent ion is dominant up to about $11.7 \mathrm{eV}$ (See Fig. 1, upper panel). From 11.7 up to $15.5 \mathrm{eV}, \mathrm{m} / \mathrm{z} 45$, formed by $\mathrm{H}$ loss of the parent ion, is by far the most intense ion. All other ions are very weak.

3) GA: This compound has been heated inside the oven of SAPHIRS up to $+100{ }^{\circ} \mathrm{C}$ to obtain a sufficient vapor pressure for the measurements; No thermal degradation was observed. No PEPICO or photoionization mass spectrometry (PIMS) work is known from the literature. We observe ions with $\mathrm{m} / \mathrm{z} 60$ (parent), 32, 31, 29 and 15. Our appearance energies for $\mathrm{m} / \mathrm{z}$ 60, 32, 31 and 29 are in good agreement with the recent electron impact ionization study (Ptasińska et al. 2005). 

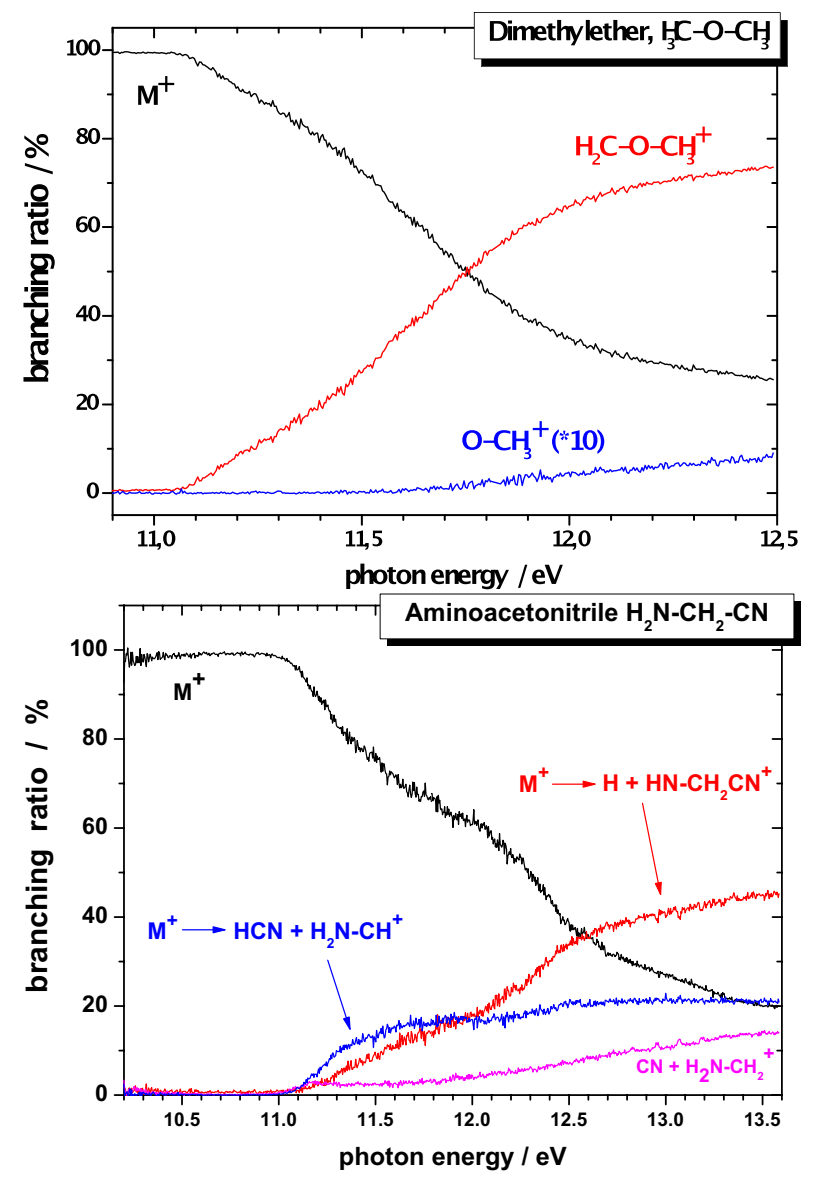

Fig. 1. Branching ratios of dissociative photoionization reactions. -(i)- Upper: Dimethylether (DIM) in the 10.9 to $12.5 \mathrm{eV}$ energy range. The parent ion (black line) is stable up to $11.1 \mathrm{eV}$ approximately. Above this energy, the dominant fragmentation reaction is loss of $\mathrm{H}$ (red line). The ion $\mathrm{m} / \mathrm{z} 31$ (blue line), corresponding to the loss of a methyl radical, is also observed in this energy range, but with weak intensity. -(ii)Lower: Amino acetonitrile (AAC) in the 10 to $13.6 \mathrm{eV}$ energy range. The parent ion (black line) is stable up to $11 \mathrm{eV}$. Above this energy, its dominant fragmentation reactions are loss of $\mathrm{H}$ (red line), HCN (blue line), and CN (magenta line), respectively.

Compared to this work, we could lower uncertainties by a factor of 10 approximately. The parent ion of GA is much less stable than the parent ion of its isomer MF. The difference between the IE of GA (IE $=10.20 \pm 0.006) \mathrm{eV}$ ) and the appearance energy of the lowest lying fragmentation channel $\mathrm{C}_{2} \mathrm{H}_{4} \mathrm{O}_{2}^{+} \rightarrow \mathrm{CH}_{2} \mathrm{OH}_{2}^{+}$ $(\mathrm{m} / \mathrm{z} 32)+\mathrm{CO}$ is only about $0.2 \mathrm{eV}$. The parent ion thus undergoes rapid ejection of a neutral $\mathrm{CO}$ molecule. In the energy range under study, the ions at $\mathrm{m} / \mathrm{z} 32$ and 31 are by far the most intense. 


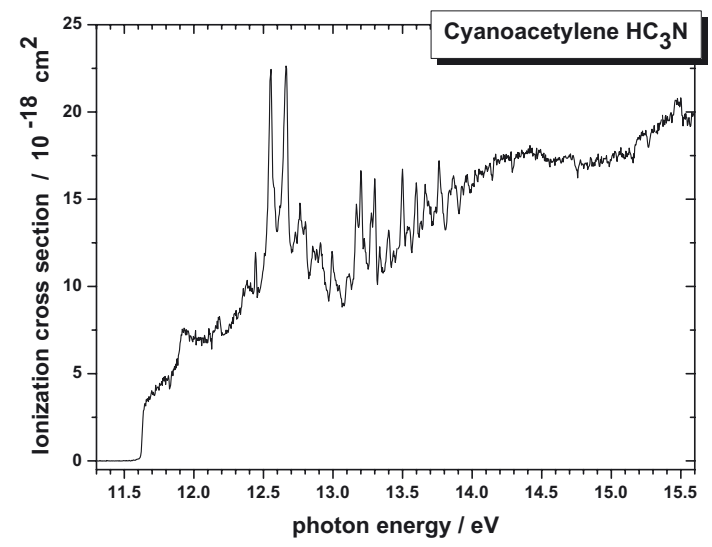

Fig. 2. Photoionization cross section of Cyanoacetylene (CA) in the 11.3 to $15.5 \mathrm{eV}$ energy range. No fragmentation reactions occur in this range due to the remarkable stability of the parent ion $\mathrm{HC}_{3} \mathrm{~N}^{+}$. The spectrum represents the PIE curve of CA calibrated using propane as a standard (see text for more details).

4) $\mathrm{AAC}\left(\mathrm{H}_{2} \mathrm{NCH}_{2} \mathrm{CN}\right)$ is a precursor of glycine by hydrolysis and has been detected in the ISM in hot cores and in giant molecular clouds such as Sgr B2 (N). It is probably formed by grain surface chemistry (Belloche et al. 2008). This is true also for MF, DIM and GA. VUV excited spectra are not found in the literature, except for an earlier photoelectron spectrum (Lacombe et al. 1989). $\mathrm{AAC}$, a liquid compound at ambient temperature was heated to about at $+40{ }^{\circ} \mathrm{C}$. Apart from its proton affinity, no gas phase ion energetics data are available in the literature. We have determined the ionization energy to be $\mathrm{IE}=(10.07 \pm 0.01) \mathrm{eV}$. The parent ion m/z 56 is stable between its IE and approximately $11 \mathrm{eV}$ where it starts to undergo fragmentation. At photon energies up to $13.6 \mathrm{eV}$, the formation of ions $\mathrm{m} / \mathrm{z} 55,30$ and 29 is observed. The branching ratios of the corresponding dissociative ionization reactions $(\mathrm{H}, \mathrm{CN}$ and $\mathrm{HCN}$ loss of the parent ion) are plotted in Figure 1 (lower panel).

5) $\mathrm{CA}\left(\mathrm{HC}_{3} \mathrm{~N}\right)$ is observed in the ISM and in the atmosphere of Titan (Teanby et al. 2007). We have studied its absorption spectrum earlier, from 5.6 to $15.5 \mathrm{eV}$ (Ferradaz et al. 2009). It is a gaseous compound at ambient temperature, which can be mixed at equal pressure with a standard of known ionization cross section. We used propane in order to convert the $\mathrm{HC}_{3} \mathrm{~N}^{+}$photoionization efficiency (PIE) spectrum into absolute ionization cross sections using the method described in Cool et al. (2005) and data on propane from Kameta et al. (2002) (see Fig. 2). Our PIE spectrum extends up to $15.5 \mathrm{eV}$ and thus, by comparison with the absorption spectrum from Ferradaz et al. (2009), we can determine the quantum yield of ionization as a function of wavelength (work in progress). CA does not at all undergo dissociative ionization in the energy range under study here, in accordance with earlier photoionization work (Okabe et al. 1973). We have determined an 
ionization energy of $\mathrm{IE}=(11.637 \pm 0.007) \mathrm{eV}$, in excellent agreement with Okabe \& Dibeler $(1973)$ who report $\mathrm{IE}=(11.64 \pm 0.01) \mathrm{eV}$. However, their PIE curve (in arbitrary units) stops at $12 \mathrm{eV}$ so that so that a comparison to our PIE spectrum at higher energies, where we observe autoionization bands, is not possible.

\section{Conclusion}

In this paper we have shown how VUV synchrotron radiation can be used to obtain high quality spectroscopic and photochemical data on interstellar molecules. We determined unknown ionization energies and fragment ion appearance energies. Branching ratios of dissociative ionization reactions are also determined. For cyanoacetylene, the ionization cross section has been measured between its IE and $15.5 \mathrm{eV}$. All these data can be used for photochemical modeling of the ISM and planetary or cometary atmospheres.

The authors wish to thank Laurent Nahon and Gustavo Garcia for excellent support during beamtime periods.

\section{References}

Belloche, A., Menten, K.M., Comito, C., et al., 2008, A\&A, 482, 179196

Butler, J.J., Holland, D.M.P., Parr, A.C., \& Stockbauer, R., 1984, Int. J. Mass Spectrom. Ion Proc., 58, 1

Carvajal, M., Margulès, L., Tercero, B., et al., 2009, A\&A, 500, 11091118

Cool, T.A., Wang, J., Nakajima, K., Taatjes, C.A., \& Mcllroy, A., 2005, Int. J. Mass Spectrom., 247, 1827

Fray, N., Bénilan, Y., Gazeau, M.C., et al., 2010, J. Geophys. Res. Planets, 115, E06010

Ferradaz, T., Bnilan, Y., Fray, N., et al., 2009, Planet. Space Sci., 57, 1022

Gaie-Levrel, F., Garcia, G.A., Schwell, M., \& Nahon, L., 2011, Phys. Chem. Chem. Phys., 13, 7024

Garcia, G.A., Soldi-Lose, H., \& Nahon, L., 2009, Rev. Sci. Instr., 80, 023102

Halfen, D.T., Apponi, A.J., Woolf, N., Polt, R., \& Ziurys, L.M., 2006, ApJ, 639, 237

Jochims, H.-W., Schwell, M., Un, S., Baumgärtel, H., \& Leach, S., 2006a, Chem. Phys., 321,159

Jochims, H.-W., Schwell, M., Un, S., Baumgärtel, H., \& Leach, S., 2006b, Chem. Phys., 321,171

Kameta, K., Kouchi, N., Ukai, M., \& Hatano, Y., 2002, J. Electron Spectrosc. Rel. Phenom., 123, 225238

Lacombe, S., Pellerin, B., Guillemin, J.C., Denis, J.M., \& Pfister-Guillouzo, G., 1989, J. Org. Chem., 54, 5958

Leach, S., Schwell, M., Un, S., Jochims, H.W., \& Baumgärtel, H., 2008, Chem. Phys., 344,147

Nishimura, T., Zha, Q., \& Meisels, G.G., 1987, J. Chem. Phys., 87, 4589

Okabe, H., \& Dibeler, V.H., 1973, J. Chem. Phys., 59, 2430 
Ptasińska, S., Denifl, S., Scheier, P., \& Tilmann, D.M., 2005, Int. J. Mass Spectrom., 243,171

Schilke, P., Benford, D.J., Hunter, T.R., Lis, D.C., \& Philipps, T.G., 2001, ApJS, 132, 281

Schwell, M., Jochims, H.-W., Baumgrtel, H., \& Leach, S., 2006, Planet. Space Sci., 54, 1073

Schwell, M., Jochims, H.-W., Baumgärtel, H., \& Leach, S., 2008, Chem. Phys., 344, 164

Schwell, M., Bénilan, Y., Fray, N., et al., 2012, Chem. Phys., 393, 107

Teanby, N.A., Irwin, P.G.J., de Kok, R., et al., 2007, Icarus, 186, 364384 